\title{
HIGH-LATITUDE CONVECTION: COMPARISON OF A SIMPLE MODEL WITH INCOHERENT SCATTER OBSERVATIONS
}

\author{
J. J. Sojka, J. C. Foster, W. J. Raitt, R. W. Schunk \\ and J. R. Doupnik
}

Center for Atmospheric and Space Sciences, Utah State University, Logan, Utah 84322

Abstract. We have compared a simple model of

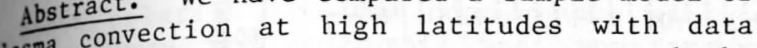
plasma convem simultaneous measurements made by obtained frent scatter facilities at Chatanika, the incoher Millstone Hill, Massachusetts in June Alaska and moderately disturbed conditions. The 1978 duringorizontal plasma drift velocities were averaged for four days to emphasize gross features of the convection pattern and reduce the effects of substorms. The convection model includes the offset of $11.5^{\circ}$ between the geographic and geomagnetic poles, the tendency of plasma to corotate about the geographic pole, and a constant dawn/dusk magnetospheric electric ffeld mapped to a circle about a center offset by $5^{\circ}$ in the anti-sunward direction from the aagnetic pole. The radius of the circle corresponds to $17^{\circ}$ of latitude and the electric potentials are aligned parallel to the noon/midnight meridian within the circle. Equatorward of the circle the potential dininishes radially and varies inversely as the fourth power of sine magnetic co-latitude. A consequence of these two offsets and the sunward alignment of the magnetospheric electric field is that our model predicts different diurnal convection patterns when viewed at different longitudes in the geographic frame. The concurrently observed diurnal distributions of horizontal plasma convection velocities are different for Chatanika and Millstone Hill even though the measurements cover approximately the sane range of magnetic latitudes. We find there is good agreement between our simple model and the gross features of these two diurnal patterns.

\section{Introduction}

In an earlier paper [Sojka et al., 1979] we presented a simple model for the dawn/dusk agnetospheric electric field mapped to the on the the flow velocities of ionospheric plasma at beth latitudes. We found that the displacement significe the geographic and geomagnetic poles the cantly affected the competition between convectition flow of the plasma and the electrive flow due to the magnetospheric fectric field. In a magnetic quasi-inertial and the with the $z$ axis parallel to the dipole axis shown sun lying in the $x-z$ plane the flow was constant be universal time (UT) independent for a the flow magnetospheric-electric field. However, in a low pattern becomes UT dependent when viewed cause of geophic inertial frame of reference bout the the rotation of the geomagnetic pole In the geographic pole.

and substs study "we have endeavored to improve Cop model by comparing model 1980 by the American Geophysical Union. predictions of plasma flow patterns with observations by the incoherent scatter radar technique. of particular interest are differences of plasma flow predicted by the model at different longitudes but otherwise at the same invariant latitude and local time. Experimental data employed were obtained by the Chatanika, Alaska, and Millstone Hill, Massachusetts, incoherent radar facilities during concurrent operations over 4 days in June 1978.

\section{Experiment Description}

Both incoherent scatter radars were operated extensively during the same 4-day period to measure the ion drift velocity vector in the $F$ region over the range of invariant latitudes $55^{\circ}$ to $76^{\circ}$ along their respective geomagnetic meridians. Chatanika, located near Fairbanks, Alaska, has a magnetic meridian pointing $29^{\circ}$ east of geographic north and sampled the region $56^{\circ}$ to $74^{\circ}$ invariant latitude $\left(55^{\circ}\right.$ to $73^{\circ}$ geographic). Millstone Hill radar has a magnetic meridian pointing $3^{\circ}$ west of geographic north and sampled the region $55^{\circ}$ to $72^{\circ}$ invariant latitude $\left(44^{\circ}-58^{\circ}\right.$ geographic). Five hours of local time separate the stations.

Both experiments utilized their very narrow beam antennas to sample the ionosphere at a succession of paired positions near their magnetic meridians. Many observations were obtained in each position by sampling at different ranges. The new 45-m steerable antenna [Evans et al., 1978] was used at Millstone Hill with excellent effect.

The local time separation between the individual samples entering into a velocity vector calculation was less than 1 hour for Chatanika and 2 hours for Millstone Hill. Maximum separation naturally occurs at the most extreme ranges and was minimized consistent with maintaining low overall statistical uncertainty of the predominant east-west flow velocity.

A set of velocity vectors could be obtained with a pair of antenna positions in about 5 minutes or less, and the same region was sampled again at about 30 minute intervals. Both Chatanika and Millstone Hill radar data were analyzed at USU (Utah State University) by using essentially the same methods. It is clearly impractical here to present error bars on the velocity vectors, but perhaps the ensemble of vectors shown will suffice. Unacceptable data were discarded by statistical signal criteria before the vectors were computed, but noisy data are evident at the extreme ranges (to the far north and south for Chatanika, and just to the far north for Millstone Hil1).

The upper panels of Figures 1 and 2 show the ion drift velocities obtained from Chatanika and Millstone Hill, respectively, for 4 days of 

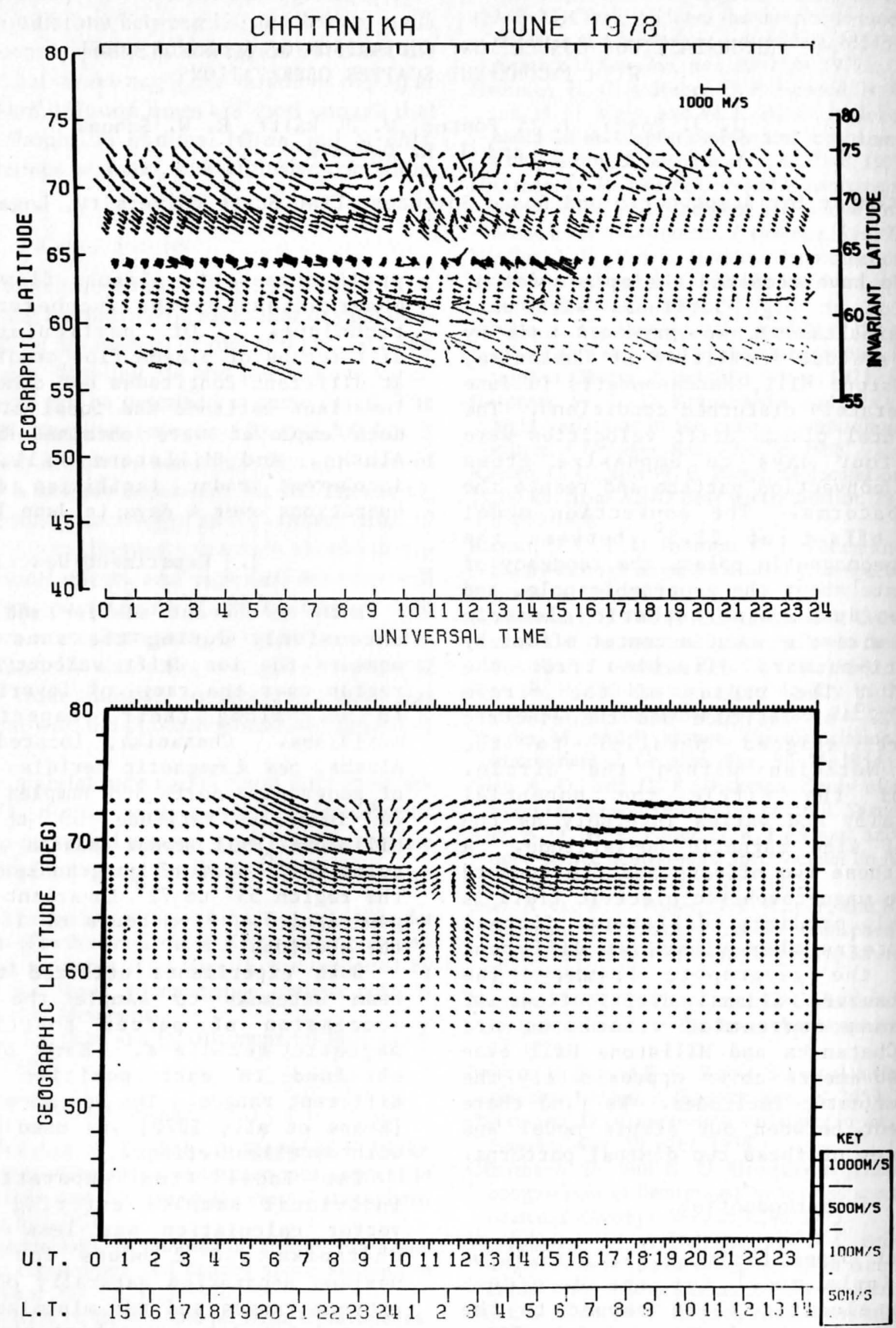

Fig. 1. Distribution of horizontal plasma drift velocities observed from Chatanika (upper panel) and the predictions for this location of our convection model (lower panel).

experiments. For the purposes of this paper the detailed measurements have been sorted into half-hour UT bins and have also been averaged over the set of 4 days. Further, although the data are shown in geographic coordinates to facilitate comparison with the models, each station's measurements covered nearly the same invariant latitudes. A vector is plotted as a dot at the central point of observation with a line indicating strength and direction.

Although the temptation is strong to consider these data as a 'snapshot' of the entire auroral convection pattern, they are really scans over the same invariant latitudes accumulated over a 24-hour period. The overall patterns nevertheless are those commonly encountered in many similar experiments. Substorms occurred which produced strong reorientations of the flow lasting for 30 minutes to over an hour and are impossible to remove from the data set simply by averaging .

\section{Comparison of Mode1 Predictions With Observations}

\section{Development of the Convection Mode1}

The initial objective of this study was to compare the simple plasma flow model of Sojka $\mathrm{flow}^{\mathrm{W}}$ al. [1979] with experimental plasma 
observations. The model assumes that the ionospheric plasma tends to corotate about the geographic pole and that the magnetospheric electric field maps into the ionosphere about the geomagnetic pole. This electric field is epresented by a uniform dawn-dusk electric field vithin a circle bounded by $75^{\circ}$ geomagnetic atitude. The magnetic field model used is that f a dipole field. Equatorward of this circle the electric potential diminishes meridionally and inversely as the fourth power of the sine of agnetic colatitude. The consequence of an offset between the geographic and geomagnetic les to introduce very marked universal time rariations which are apparent when observing the plasma flow in the geographic frame at different longitudes yet at the same local time and magnetic latitude.

The predicted plasma flow pattern for Chatanika is shown in Figure 3 for this simple convection model. The display format was chosen to match that of the data (Figure 1, upper panel). This model reproduces only the gross characteristics of the observed ion drift pattern; namely, the westward and eastward flow directions in the local evening and morning sectors (the zonal flow-pattern) and the west-south-east reversal around midnight. The most obvious feature of the experimental data is the movement of the convection field to low latitudes at night and its retreat to high latitudes in the day, a feature which is absent
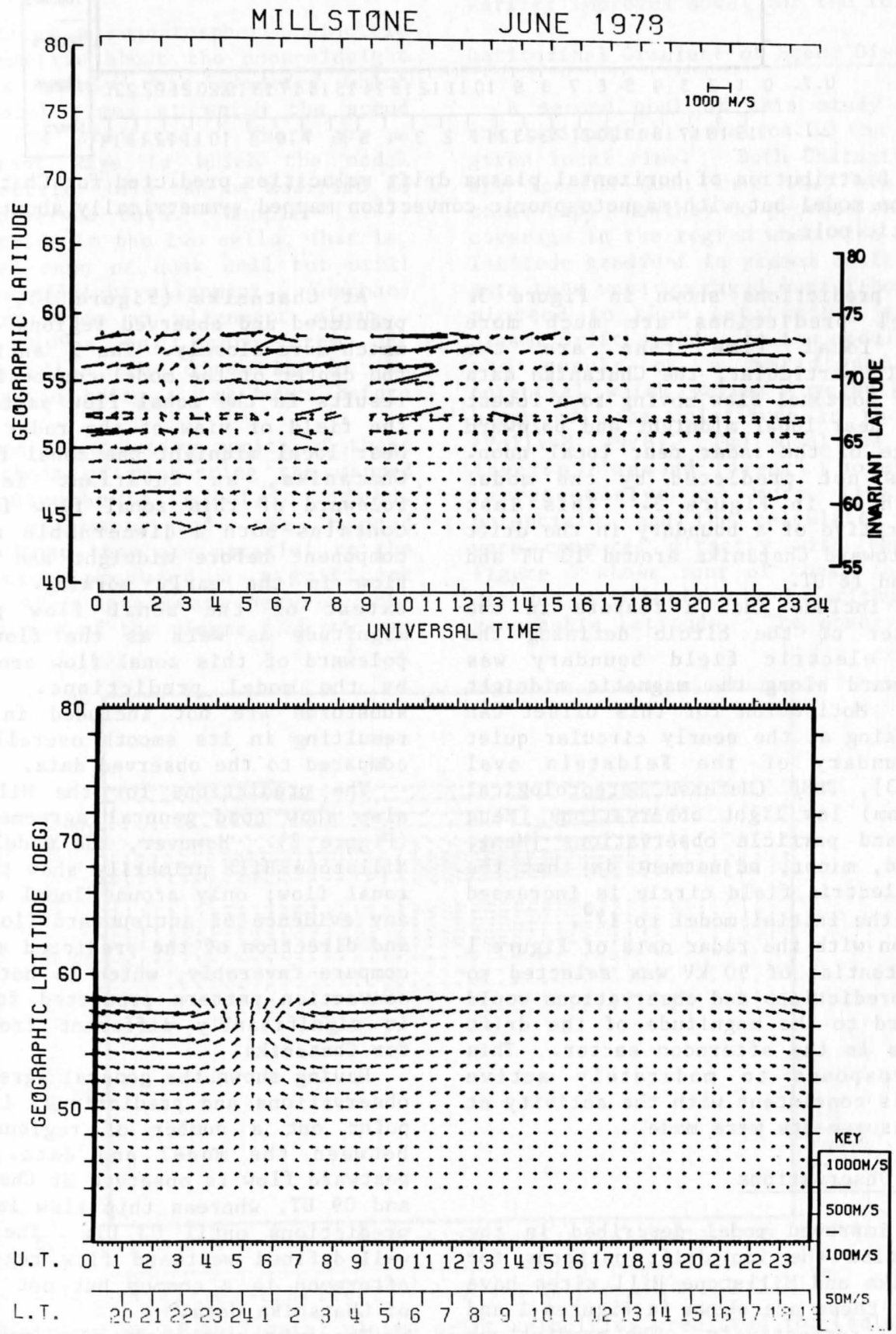

Fig. 2, Distribution of horizontal plasma drift velocities observed from Millstone Hill (upper panel) and the predictions for this location of our convection model (lower panel). 


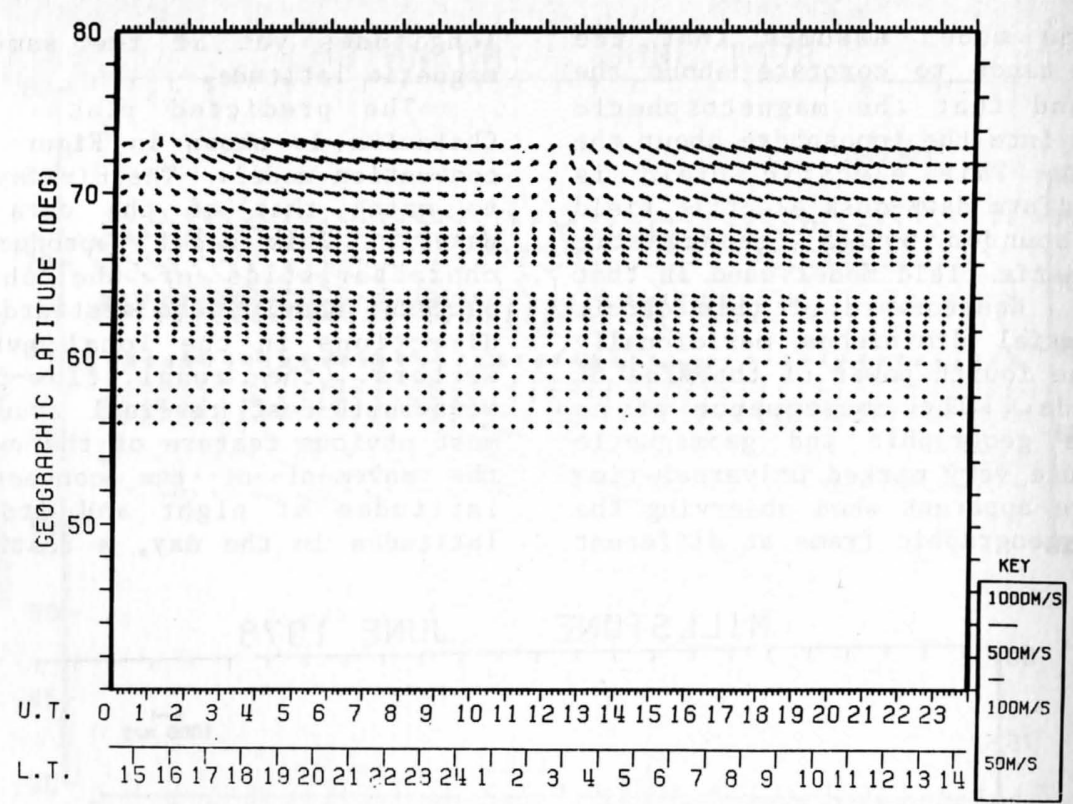

Fig. 3. Distribution of horizontal plasma drift velocities predicted for Chatanika by our convection model but with magnetospheric convection mapped symmetrically about the geomagnetic pole.

from the model predictions shown in Figure 3. Also the model predictions are much more symmetric in local time than are the observations. In particular, the Chatanika data show a region of nonzonal flow moving to a lowest latitude of $68^{\circ}$ near local midnight and poleward out of the range of the radar near local noon. This region is not predicted by the model calculations shown in Figure 3. This last feature is indicative of a boundary in the drift pattern moving toward Chatanika around 10 UT and away again around $18 \mathrm{UT}$.

In order to include such a feature in the model the center of the circle defining the magnetospheric electric field boundary was shifted antisunward along the magnetic midnight meridian by $5^{\circ}$. Motivation for this offset can be found by looking at the nearly circular quiet time inner boundary of the Feldstein oval [Feldstein, 1963], DMSP (Defense Meteorological Satellite Program) low light observations [Meng et al., 1977] and particle observations [Meng, 1979]. A second, minor, adjustment is that the radius of the electric field circle is increased from the $15^{\circ}$ of the initial model to $17^{\circ}$.

For comparison with the radar data of Figure 1 a cross tail potential of $90 \mathrm{kV}$ was selected so that the model predictions and observations would agree with regard to the magnitude of the drift velocity vectors in the afternoon sector. This potential corresponds to moderately active conditions and is consistent with the activity at the time the measurements were made.

\section{Comparison With Observations}

By using the improved model described in the previous subsection the ion drift patterns for both the Chatanika and Millstone Hill sites have been calculated; these are shown in Figures 1 and 2 (lower panels), respectively. The predictions of the improved model are seen to be in good qualitative agreement with the observations.
At Chatanika (Figure 1), features of the predicted and observed regions of zonal flow now match more closely. The $5^{\circ}$ antisunward of fset of the center of the model convection electric field results in the zonal flow pattern moving out of the field of view of the radar near local noon. Near local midnight the zonal flow is closest to Chatanika, an invariant latitude of $65^{\circ}$. Poleward of this zonal flow is a region which contains both a discernable antisunward flor component before midnight and a reversed zonal flow in the early morning. The latitudinal extent of the zonal flow pattern and its magnitude as well as the flow characteristics poleward of this zonal flow are well represented by the model predictions. The effects of substorms are not included in the model, thus resulting in its smooth overall appearance when compared to the observed data.

The predictions for the Millstone Hill site also show good general agreement with the data (Figure 2). However, the model predictions for Millstone Hill primarily show the main region of zonal flow; only around local midnight is there any evidence of antisunward flow. The magnitude and direction of the predicted and observed flows compare favorably, which is noteworthy since the convection pattern predicted for Millstone Hill is significantly different from that predicted for Chatanika.

Having shown the general agreement between the observations and predictions, it is necessary to point out a number of regions of discrepanc. between the model and data. The afterno 22 westward flow is observed at Chatanika between the and 09 UT, whereas this flow is not seen in the predictions until 03 UT. The occurrence of well-defined westward flow region in the eature afternoon is a common but not universal of Chatanika data.

The west-south-east flow reversal near locans midnight occurs in the vicinity of the pattern. discontinuity in the ionospheric current patte 
weling of the Harang discontinuity requires odeling ocalized asymmetric potential distributions rery local and Matsushita, 1979], and even then Ramide ant is only obtained in restricted regions. 1 reement used here does not attempt to include the model uiled physics associated with the Harang the detailuity. Thus the general comparison of the iscontions and predictions in this local time gor is good even though a precise account of fine structure is not to be expected.

both Chatanika and Millstone Hill the redicted flow reversal near midnight appears hetween 1 and 3 hours later than observed, and at chanika the noon sector pattern is predicted to occur several hours later than seen in the data. There appears to be a systematic time slippage theen the predictions of the model and the data.

The model contains a magnetospheric electric field that is symmetric about the noon-midnight eridian. It is mainly this symmetry which determines the local times at which the speed reversal regions are predicted. There are a unber of different ways in which the model sagnetospheric electric field can be modified to better fit the observed data. Heppner [1977] introduces asymmetries in the two cells, that is, having an enhanced dawn or dusk cell but still asintaining a noon-midnight alignment. Yasuhara et al. [1975] introduce an alignment along a aeridian offset by a few hours from the noon-midnight meridian, the direction of the offset on the dayside being a few hours before aidday.

We have examined the relative merits of these two empirical methods of distorting the mapped cross-tail magnetospheric electric field. ddjusting the alignment of the mapped equipotentials so that they are parallel to the 10-22 LT direction produced a significant inprovement in the agreement between the predicted distribution of the plasma flow vectors and those observed at Chatanika, whereas the other method failed to improve the agreement. Figure 4 shows the predictions of a model identical to that used earlier, except for a skewing of the magnetospheric electric field to give equipotentials parallel to the 10-22 LT direction. Better agreement between this model and the Chatanika measurements is seen in the postnoon region. Also the timing of the flow reversals near local noon and midnight is in better agreement with the measurements. This figure is presented as an indication of how empirical adjustments can be used to provide a better match to the data. We are reluctant to recommend the use of this empirical feature at this stage because of the limited data base used in the comparison, and we revert back to the earlier improved model for the following section.

\section{Latitudinal Gradient of Speed Distribution}

A second goal of this study was to estimate the latitudinal variation of the flow speed at a given local time. Both Chatanika and Millstone are located such that they see little of the polar cap, but they do provide excellent latitude coverage in the region where the model predicts a latitude gradient in plasma drift speed. The two data sets were averaged over 1-hour intervals and plotted to show latitudinal gradient of the plasma drift speed. In the model the gradient is the result of a meridionally diminishing potential which varies inversely as the fourth power of sine colatitude in the magnetic frame [Volland, 1978]. The model was also run for an inverse 'second power' potential gradient representative of more slowly diminishing potentials, and the calculated speed gradients were. compared directly with the plotted data. Figure 5 shows four of these latitude gradients derived by plotting the logarithm of speed versus geographic latitude. The observations shown are

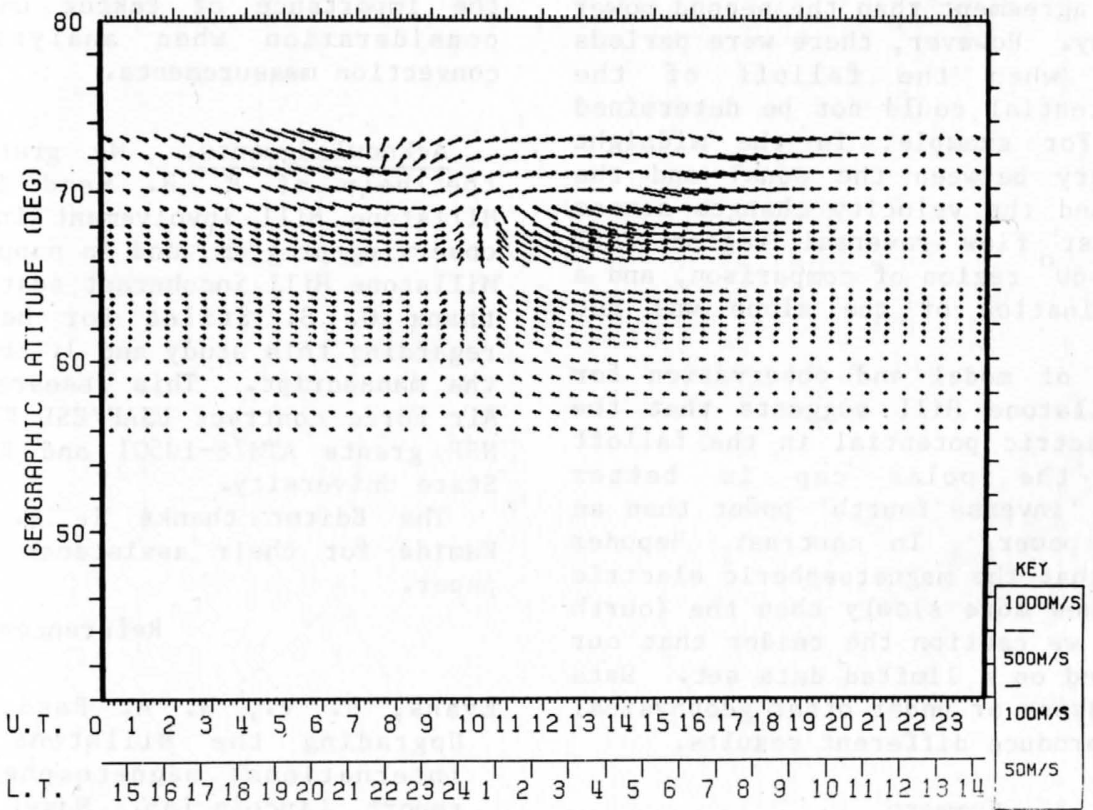

Fig. 4. Distribution of horizontal plasma drift velocities predicted for Chatanika by our convection model modified to include an empirical skew to the mapped magnetospheric electric field resulting in equipotentials aligned parallel to the 10-22 LT direction. 

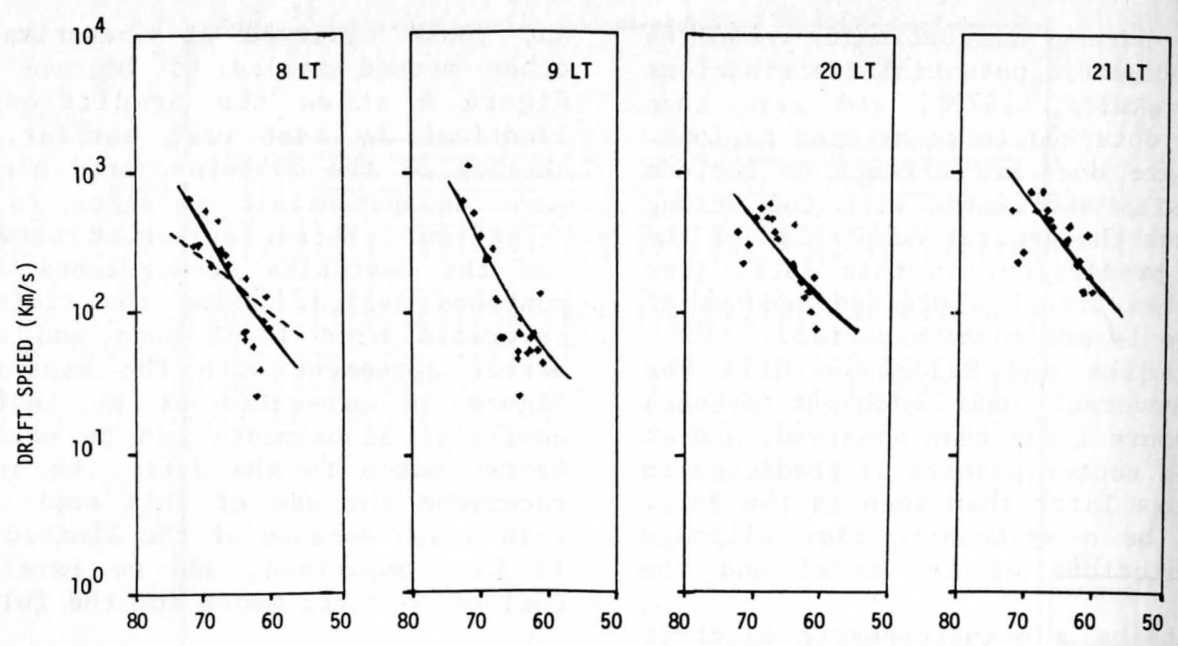

GEOGRAPHIC LATITUDE (deg.)

Fig. 5. Variation of plasma drift speeds with geographic latitude for four local time periods as observed at Chatanika (data points). The data were collected over a time period of 1 hour immediately prior to the time shown. The solid lines are the model predictions for the indicated local times. The dashed line corresponds to the variation in drift speed for an inverse second power decay in the magnetospheric electric potential (see text).

all from Chatanika, the data being represented by points and the 'fourth power' model gradient being represented by the solid lines. In the first panel of Figure 5 a dashed line is included; this represents a 'second power' model gradient.

It is apparent that the 'fourth power' model gradient clearly produces better agreement with the measurements than the 'second power' gradient. The four local times shown in Figure 5 were chosen for display because a smooth gradient in the speed distribution was clearly evident in the data at these times. A similar comparison between model predictions and observations at other local times indicated that the fourth power produced a better agreement than the second power for most of the day. However, there were periods during the day when the falloff of the magnetospheric potential could not be determined unambiguously. For example, in the midnight sector the boundary between the zonal and the polar cap flows and the velocity changes across the west-south-east flow reversal region fall within the $72^{\circ}$ to $60^{\circ}$ region of comparison, and a meaningful determination of the slope was not possible.

The comparison of model and observation for Chatanika and Millstone Hill suggests that the magnetospheric electric potential in the falloff region outside the polar cap is better represented by an 'inverse fourth' power than an 'inverse second' power. In contrast, Heppner [1977] suggested that the magnetospheric electric potential diminishes more slowly than the fourth power. However, we caution the reader that our conclusion is based on a limited data set. Data taken at other seasons or under other geophysical conditions could produce different results.

\section{Summary}

The gross features of the diurnal variation of horizontal plasma drift velocities measured concurrently by the incoherent scatter radar installations at Chatanika and Millstone Hill in June 1978 have been compared with the predictions of a simple model of ionospheric convection. The model used is based on that of Sojka et al. [1979] and refined by offsetting the mapped magnetospheric electric potential $5^{\circ}$ from the magnetic pole in the antisunward direction. We have shown that the differences in the convection pattern observed at the two sites are consistent with the predictions of this simple model. We are encouraged by the agreement between the model and the data and feel that further comparisons would be fruitful in refining the model. The comparison we have made in this study underlines the importance of taking universal time into consideration when analyzing high-latitude convection measurements.

Acknowledgments. We gratefully acknowledge the help of R. H. Wand in scheduling the Millstone Hill involvement in the collaborative observing program, and in supplying data from the Millstone Hill incoherent scatter radar. We also thank G. S. Stiles for helpful discussions regarding this study and.J. Evans for comments of the manuscript. This research was supported by Air Force contract USAF/ESD F19628-79-C-0025 and NSF grants ATM78-10501 and ATM78-05747 to Utal State University.

The Editor thanks R. A. Greenwald and Y. Kamide for their assistance in evaluating this paper.

\section{References}

Evans, J. V., W. A. Reed, and P. Stetson, Upgrading the Millstone Hill radar final international magnetosphere studies, flnol., report, Lincoln Lab., Mass. Inst. of Technol. Lexington, Mass., 1978.

Feldstein, V. I., Some problems concerning the morphology of auroras and magnetic disturb 
thigh 1atitudes, Geomagn. Aeron., 3, 183, 1963. J. P., Empirical models of geppner, high-latitur-1125, 1977.

Res. 82 Y., and S. Matsushita, Simulation a aide, of ionospheric electric fields and currents in relation to field-aligned currents, Substorms, J. Geophys. Res., 84, 2, $99-4115,1979$.

40.-I., Diurnal variation of the auroral oval size, J. Geophys. Res., 84, 5319-5324, 1979.

Neng, C.-I., R. H. Holzworth, and S.-I. Akasofu, Auroral circle - Delineating the poleward boundary of the quiet auroral belt, J. Geophys. Res., 82, 164-172, 1977.
Sojka, J. J., W. J. Raitt, and R. W. Schunk, Effect of displaced geomagnetic and geographic poles on high-latitude plasma convection and ionospheric depletions, J. Geophys. Res., 84, 5943-5951, 1979.

Volland, H., A model of the magnetospheric electric convection field, J. Geophys. Res., 83, 2695-2699, 1978.

Yasuhara, F., Y. Kamide, and S.-I. Akasofu, Field-aligned and ionospheric currents, Planet. Space Sci., 23, 1355-1368, 1975.

(Received July 5, 1979;

revised September 25, 1979;

accepted September 28, 1979.) 